\title{
Author Index to Volume 21 - 1994
}

Adams, C.: 203 (Aug) Agranovish, A.: 306 (Nov) Aicardi, J.: 185 (Aug) Alessandro, B.: 266 (Aug) Anderson, J.: 203 (Aug) Andreula, C.: 266 (Aug) Ang, L.C.: 306, 350 (Nov) Archibald, C.J.: 9 (Feb) Ashby, P.: 125 (May) Assis, L.P.A.: 252 (Aug) Attig, E.: 15 (Feb)

Badrising, U.A.: 53. 57 (Feb) Barbieri, F.: 29 (Feb) Barnett, H.J.M.: 85 (May) Basinski, A.: 125 (May) Battershill, J.H.: 78 (Feb) Becker, L.E.: 273 (Aug) Becker, W.J.: 285 (Aug) Begley, H.: 306 (Nov) Benoit, B.G.: 72 (Feb) Bergeron, C.: 311 (Nov) Bernstein, M.: 213 (Aug) Berry, H.: 38 (Feb) Bertolasi, L.: 325 (Nov) Bissonette, D.J.: 137 (May) Blume, W.T.: 252 (Aug) Bolton, C.F.: S28 (Suppl 1) Botez, M.I.: 353 (Nov) Botez-Maquard, T.: 353 (Nov) Boulton, A.J.M.: S18 (Suppl 4) Bril, V.: 34 (Feb); S8 (Suppl 4) Brooke, M.H.: 120 (May) Bruni, J.: S3 (Suppl 3) Burnham, W.M.: 3 (Feb)

Coyne, T.J.: 129 (May) Cabantog, A.M.: 213 (Aug) Camfield, C.: S7 (Suppl 3) Camfield, P.: S7 (Suppl 3) Capparelli, G.: 29 (Feb) Carlo, P.: 266 (Aug) Carpio, A.: 43 (Feb) Chang, Y-Y.: 271 (Aug) Chiappa, K.H.: S12 (Suppl I) Chudley, A.: 203 (Aug) Chung, D-G.: 104 (May) Ciccarelli, A.: 29 (Feb)

Clarke, D.B.: 64 (Feb) Claudio, G.: 266 (Aug) Crisci, C.: 29 (Feb) Curran, T.: 311 (Nov) Cwik, V.A.: 120 (May)

Darwish, H.Z.: 203 (Aug) da Silva, M.C.: 299 (Nov) Davey, P.: 319 (Nov) De Grandis, D.: 325 (Nov) Degroot, W.R.: 203 (Aug)
Del Bigio, M.R.: 299 (Nov)

Drake, J.M.: 299 (Nov)

Dykes, R.W.: 233 (Aug)

Eastwood, M.R.: 358 (Nov)

Ebly, E.M.: 33I (Nov)

Enrico, M.: 266 (Aug)

Escobar, A.: 43 (Feb)

Espinosa, J.A.: 14I (May)

Farmer, J-P.: 64 (Feb)

Fayez, N.A.: 72 (Feb)

Feldman, E.L.: S3 (Suppl 4)

Findlay, J.M.: 67 (Feb); 146 (May); 278 (Aug)

Fisk, J.D.: 9 (Feb)

Follis, F.: 227 (Aug)

Forstl, H.: 78 (Feb)

Francesco, D.: 266 (Aug)

Francis, Ô.: 3 (Feb)

Frecker, M.F.: 112 (May)

Freedman, M.: 104 (May)

Georgiev, D.: 60 (Feb)

Gordon, B.A.: 252 (Aug)

Greenberg, M.: 273 (Aug)

Hachinski, V.: 358 (Nov)

Hay, R.: 287 (Aug)

Ho, L.T.: 194 (Aug)

Hogan, D.B.: 331 (Nov)

Hospodar, M.: 133 (May)

Hudoba, P.: 319 (Nov)

Ichise, M.: 104 (May)

Jaoquin, A.J.: 72 (Feb)

Jiang, F.: 100 (May)

Jog, M.S.: 38 (Feb)

Johnson, E.: 233 (Aug)

Kessler, R.: 227 (Aug)

Kertesz, A.: 358 (Nov)

Kirk, A.: 350 (Nov)

Klein, G.M.: 262 (Aug)

Konasiewicz, S.J.: 219 (Aug); S17

(Suppl I)

Kondziolka, D.: 137 (May)

Kreeft, J.H.: 252 (Aug)

Kuhn, S.: 203 (Aug)

Lacson, A.G.: 203 (Aug)

Lalonde, R.: 353 (Nov)

Lang, A.E.: 311 (Nov)

Li, C-X.: 233 (Aug)

Liauw, L.: 53, 57 (Feb)

Liu, J-S.: 271 (Aug)

Loembe, P.M.: 339 (Nov)

Lohuis, N.A.: 252 (Aug)

Loring, D.W.: 346 (Nov)

Lowry, R.B.: 203 (Aug)
Lunsford, L.D.: 137 (May)

Miller, K.: 227 (Aug)

Madera, A.: 43 (Feb)

Majumdar, R.: 120 (May)

Manganotti, P.: 325 (Nov)

Maria, T.: 266 (Aug)

Massimo, G.: 266 (Aug)

McCandlish, C.A.: 233 (Aug)

Meador, K.J.: SI2 (Suppl 3); 346 (Nov)

Meldrum, H.: 85 (May)

Mielke, B.W.: 67 (Feb)

Mihic, S.J.: 3 (Feb)

Milanov, I.: 60 (Feb)

Montes, J.L.: 64 (Feb)

Mortilla, M.: 248 (Aug)

Moulton, R.J.: S17 (Suppl 1): 219

(Aug)

Muizelaar, J.P.: S6 (Suppl 1)

Muller, M.: 273 (Aug)

Murray, M.: 24 (Aug)

Murray, T.J.: 9 (Feb)

Nag, S.: 24 (Feb)

Nichols, M.E.: 346 (Nov)

O'Brien, P.F.: 319 (Nov)

O'Connor, P.: S17 (Suppl 1)

Oladehin, A.: 233 (Aug)

Oppenheimer, S.R.: 252 (Aug)

Osimani, A.: 104 (May)

Pett, S.: 227 (Aug)

Paredes, V.: 43 (Feb)

Parhad, I.: 358 (Nov)

Parhad, I.M.: 33। (Nov)

Pauwels, E.K.J.: 53 (Feb)

Perry, J.R.: 34 (Feb)

Phillips, S.: 358 (Nov)

Placencia, M.: 43 (Feb)

Plewes, M.: 306 (Nov)

Pogue, J.M.: 104 (May)

Polo, A.: 325 (Nov)

Pontefract, A.: 9 (Feb)

Preul, M.C.: 141 (May)

Provias, J.: 273 (Aug): 311 (Nov)

Pryse-Phillips, W.E.M.: 112 (May)

Racine, R.: 100 (May)

Ramani, R.: 319 (Nov)

Rewcastle, B.: 358 (Nov)

Riopelle, R.J.: 24 (Feb)

Ritvo, P.G.: 9 (Feb)

Rizzuto, N.: 325 (Nov)

Robitaille, Y.: 141 (May)

Rockwood, K.: 358 (Nov)

Roos, R.A.C.: 53, 57 (Feb)

Ropper, A.H.: S23 (Suppl 1)

Rose, M.S.: 262 (Aug)

Rouleau, G.: 64 (Feb)
Russell, N.A.: 72 (Feb)

Rutka, J.T.: 273 (Aug)

Scremin, O.U.: 227 (Aug)

Santangelo, R.: 29 (Feb)

Santillàn, F.: 43 (Feb)

Sarnat, H.B.: 203 (Aug)

Schròder, M.L.: S6 (Suppl 1)

Schwartz, M.L.: 319 (Nov)

Scott, T.: 133 (May)

Seland, T.P.: 262 (Aug)

Seshia, S.S.: 203 (Aug)

Sharpe, J.A.: 125 (May)

Shedden, P.M.: 219 (Aug)

Shul, D.: 306 (Nov)

Sima, A.: S23 (Suppl 4)

Sima, A.A.F.: S13 (Suppl 4)

Smith, S.L.: 273 (Aug)

Sneddon, W.B.: 3 (Feb)

St. George-Hyslop, P.H.: 248 (Aug)

Stefania, M.: 266 (Aug)

Stefano, B.: 266 (Aug)

Strong, H.R.: I12 (May)

Suchowersky, O.: 48 (Feb)

Tampieri, D.: 141 (May)

Tan, L.: 306 (Nov)

Thierer, D.E.: 331 (Nov)

Thrasher, D.: 295 (Nov)

Tsai, T-C.: 27 ( Aug)

Tuor, U.I.: 299 (Nov)

Turley, J.E.: 38 (Feb)

Turnbull, J.: 100 (May)

Ukachoke, C.: 125 (May)

Vaula, G.: 248 (Aug)

Victor, M.: 88 (May)

Vielvoye, G.J.: 57 (Feb)

Vielvoye, J.: 53 (Feb)

Wallace, M.C.: 129 (May)

Wernly, J.: 227 (Aug)

Wapenski, J.: 133 (May)

Waters, C.H.: 259 (Aug)

Waters, R.S.: 233 (Aug)

Watters, G.V.: 64 (Feb)

Weikers, N.: 133 (May)

Wells, G.A.: 252 (Aug)

Wilder, B.J.: S17 (Suppl 3)

Wintzen, A.R.: 53, 57 (Feb)

Wu, H-S.: 271 (Aug)

Young, C.S.: 319 (Nov)

Young, G.B.: S3 (Suppl I); 252 (Aug); 295 (Nov)

Zanette, G.: 325 (Nov)

Zee, D.S.: 177 (Aug)

Zochodne, D.W.: 24 (Feb); 194 (Aug) 